\title{
Longitudinal Aerodynamic and Stability Characteristics of High-Aspect-Ratio Unmanned Aerial Vehicles in Gust Response
}

Junli Wang1*, Wensheng Zhang1, Bolin Feng1, Zhigui Ren¹, Qinghe Zhao²

Wang J (iD https://orcid.org/0000-0001-8666-012X Zhang W (D) https: //orcid.org/0000-0001-9875-2304

Feng B (D) https://orcid.org/0000-0002-4687-0824

Ren Z (D) https://orcid.org/0000-0002-5597-8095

Zhao Q iD https://orcid.org/0000-0003-1509-3425

\section{How to cite}

Wang J; Zhang W; Feng B; Ren Z; Zhao Q (2019) Longitudinal Aerodynamic and Stability Characteristics of High-Aspect-Ratio Unmanned Aerial Vehicles in Gust Response. J Aerosp Technol Manag, 11: e2719. https://doi.org/10.5028/jatm.v11.1052

ABSTRACT: The aim of this work is to propose an accurate and reliable numerical simulation method of gust response, so as to analyze longitudinal stability characteristics of high-aspect-ratio unmanned aerial vehicles (UAVs) under gust response. Based on the dual-time stepping method, the unsteady Navies-Stokes equation was solved. By introducing grid velocity to study the effects of gust, the numerical simulation of gust response was realized. Moreover, the numerical simulation method was verified to be accurate by using the theoretical value and reference value obtained in previous research. The calculation results of the high-aspect-ratio UAV under the 1-cos gust reveal that longitudinal aerodynamic forces of high-aspect-ratio UAVs changed. In the whole range of gust gradient length, the UAVs were always in the state of static stability. However, with the increase of gust velocity, static stability margin decreased. The numerical simulation method of gusts established in this study preferably overcomes the possible numerical oscillations and divergence problems caused by excessive gust velocity. The analysis on longitudinal static stability and stability margin of high-aspect-ratio UAVs under the effects of gusts can ensure flight quality and safety of UAVs under the effects of gusts.

KEYWORDS: High-aspect-ratio UAVs, Gust response, Numerical simulation, Longitudinal stability characteristics.

\section{INTRODUCTION}

In the design of modern unmanned aerial vehicles (UAVs), the influences of various inevitable special environments on UAVs have to be taken into account, such as the dynamic response of UAVs in gust areas. When UAVs encounter a gust, the UAV body bears large unsteady aerodynamic loads, resulting in structural deformation and rigid body motion of UAVs. In addition, too large amplitude of gusts can cause problems such as difficult operation of UAVs, fatigue damage of structures and static instability. For manned aircrafts, pilots can operate the control plane according to the detection data, so as to reduce gust loads. For highaspect-ratio UAVs, gust loads greatly affect longitudinal stability, aeroelasticity and aerodynamic noise due to light weight and large flexibility of wings. In the design of UAVs, aerodynamic load response of high-aspect-ratio UAVs when encountering gusts needs to be accurately simulated. In accordance with the strength of gust loads, different procedures of load reduction of gusts are preset to reduce gust loads when UAVs fly through gusts, thus ensuring flight safety.

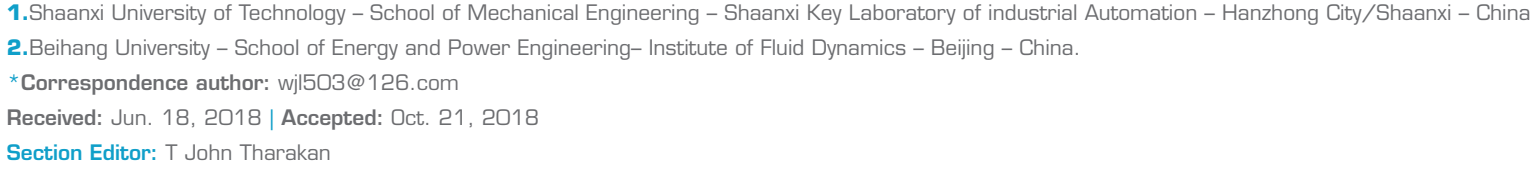


In the last few decades, scholars have conducted a lot of researches on gust response of aircrafts by using the experimental and numerical simulation methods. The early study of aircraft gust response was mainly performed in the frequency domain by using the linear plate aerodynamic force and combining with aeroelastic equation (Wu et al. 2010). With the development of computational fluid dynamics, the gust response of aircraft was calculated by solving the unsteady Euler and Navier-Stokes equations in the time domain (Singh and Baeder 1997; Zhan and Qian 2007; Gu et al. 2013; Yang and Obayashi 2004). Later, the reduced-order model method based on CFD emerged and the gust response of aircraft was calculated by employing the reducedorder model (Zaide and Raveh 2006; Gennaretti and Mastroddi 2004; Zhang et al. 2009).

In recent years, many scholars have paid more attention to the researches on numerical simulation of gust response and gust load alleviation of high-aspect-ratio UAVs. Researches mainly focused on the time-domain numerical simulation method of gust response of high-aspect-ratio flexible aircraft. The scholars also studied the influence of large-aspect-ratio flexible aircraft gust response on elastic deformation and fluttering speed. Currently, studies mainly focus on the influence of gust alleviation devices on the gust response and free flutter speed of high-aspect-ratio flexible aircraft. In addition, in view of the model of highaspect-ratio flexible aircraft, gust alleviation control laws were designed and the gust alleviation results for random gusts were studied (Liu et al. 2016; Guo et al. 2017; Patil and Taylor 2006; Shao et al. 2010; Tang et al. 2010). Research results in recent years demonstrate that existing studies on gust response of high-aspect-ratio UAVs mainly focus on gust simulation methods and gust load alleviation, while the influences of gust loads on longitudinal stability characteristics of high-aspect-ratio UAVs were rarely investigated. By utilizing the dual-time stepping method, this study solved the unsteady Navies-Stock equation. By referring to ideas in references (Parameswaran and Baeder 1997) and introducing grid velocity into the study to reveal the effects of gusts, this research conducted the numerical simulation on gust response of high-aspect-ratio UAVs and investigated the influences of gust loads on longitudinal stability of UAVs.

\section{METHODOLOGY \\ GOVERNING EQUATION}

The governing equation in the study was the unsteady Navier-Stokes equation in 3D integral form and its expression under Cartesian inertial coordinate system is shown as follows (Eq. 1) (Gilmanov and Sotiropoulos 2005; Orang 2014; Wang 2011):

$$
\begin{aligned}
& \frac{\partial}{\partial t} \iiint_{\Omega} \bar{Q} d \Omega+\iint_{S}\left(\bar{G}-\bar{Q}_{\vec{q}}\right) \cdot d \vec{S}=\frac{1}{\operatorname{Re}} \iint_{S} \bar{F}^{V} \cdot d \vec{S} \\
& \bar{Q}=\left[\begin{array}{c}
\rho \\
\rho u \\
\rho v \\
\rho w \\
\rho e_{t}
\end{array}\right], \bar{G}=\left[\begin{array}{ccc}
\rho u & \rho v & \rho w \\
\rho u^{2}+p & \rho v u & \rho w u \\
\rho u v & \rho v^{2}+p & \rho w v \\
\rho u w & \rho v w & \rho w^{2}+p \\
\rho u h_{t} & \rho v h_{t} & \rho w h_{t}
\end{array}\right] \\
& \vec{q}_{b}=u_{b} i+v_{b} j+w_{b} k, \quad \bar{F}^{V}=\left[\begin{array}{ccc}
0 & 0 & 0 \\
\tau_{x x} & \tau_{y x} & \tau_{z x} \\
\tau_{x y} & \tau_{y y} & \tau_{z y} \\
\tau_{x z} & \tau_{y z} & \tau_{z z} \\
\varphi_{x} & \varphi_{y} & \varphi_{z}
\end{array}\right]
\end{aligned}
$$


where $\bar{Q}$ is the flow field variable; $\bar{G} \bar{F}^{v}$ are the non-viscosity convection term and viscosity dissipation terms, respectively; $\vec{q}_{b}$, $\Omega, S$ and $\overrightarrow{d S}$ are the velocity of mesh motion in computing domain, the control volume, the surface of control volume and the outer normal area vector of $S$ infinitesimal, respectively.

\section{NUMERICAL SIMULATION METHOD}

By using the finite volume method, the numerical solution of Eq. 1 was obtained. The discretization of unsteady time derivative terms was carried out by using the dual time-stepping method, and the implicit LU-SGS approximation method was utilized for pseudo-time stepping method (Gong et al. 2016; Song et al. 1999). This time-stepping method had two-order accuracy, took up less memory and had higher computational efficiency. Moreover, the discretization of space convection term and viscosity term were carried out by using Roe flux difference and second order upwind, respectively. The spatial discrete method had second-order accuracy, and the Van Albada limiter was employed to suppress numerical oscillations near the shock during the calculation (Van Albada et al. 1982). By taking an example of an aircraft encountering step-type gusts in the angle of attack (as shown in Fig. 1), the aircraft flying horizontally at the speed of $U_{\infty}$ in the initial state were suddenly affected by vertical upward gust $w_{g}$, which equaled to increasing the angle of attack suddenly by $\Delta \alpha\left(\Delta \alpha=\operatorname{arctg}\left(w_{g} / v_{\infty}\right)\right)$. If the boundary condition of an angle of attack's sudden change was directly applied to the aircraft, numerical oscillation appeared in the calculation when this sudden change value was large, resulting in instability and divergence of calculation results. In addition, when applying the boundary condition of an angle of attack's sudden change to the aircraft, not only the angle of attack of the aircraft suddenly changed, but also pitch angle velocity was generated through coupling. Therefore, the calculated response is not the independent response of the angle of attack's sudden change.

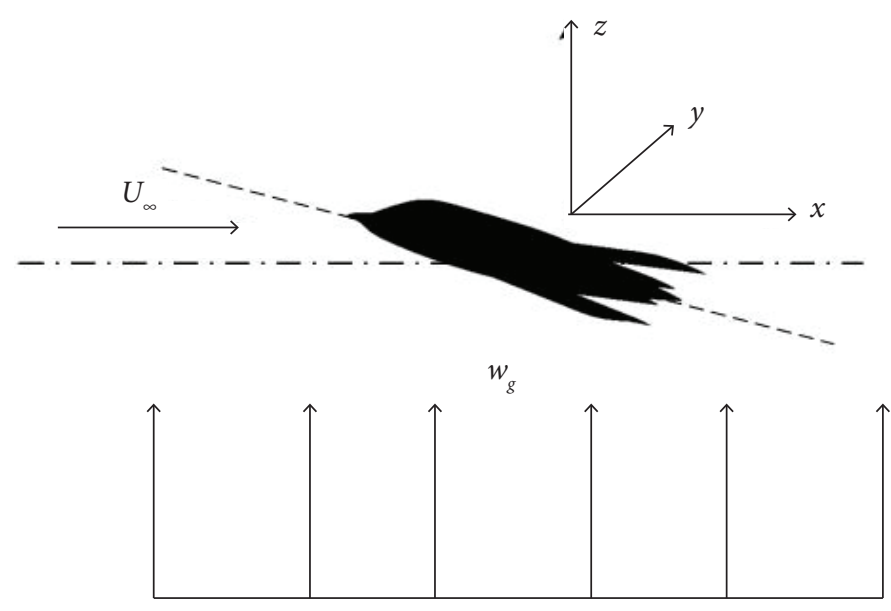

Figure 1. Sketch of gust leading a step change in the angle of attack.

Therefore, by introducing the concept of grid velocity into the study, when the aircraft was affected by vertical upward gusts, it was equivalent to the condition that there was a suddenly increasing vertical grid velocity in the whole flow field, and there was no additional pitch movement. According to the idea of relative movement, if the movement velocity of grids was $w_{g}$, it indicated that the whole computing domain moved downward at the speed of $-w_{g}$ on conditions that the grid did not move. In the governing equation, this is specifically expressed as follows (Eq. 2):

$$
\begin{aligned}
& \vec{q}_{b}=u_{b} i+v_{b} j+w_{b} k \\
& u_{b}=0 、 v_{b}=0 、 w_{b}=-w_{g}
\end{aligned}
$$


By substituting Eq. 2 into Eq.1 for unsteady flow field calculation, the gust response history of the aircraft under the effects of gusts was obtained.

\section{VERIFICATION}

In view of step change gusts in the angle of attack shown in Fig. 1, the gust numerical simulation method was verified by using the NACA0006 airfoil. The $C$ mesh in the dimension of $181 \times 31$ was used as the computation mesh, the distance from the grids in the first layer near the wall to the wall surface was $1 \times 10^{-5}$, and the free stream Mach numbers were $M_{\infty}=0.3,0.5,0.65,0.8$. The initial angle of attack was $\alpha=0$, and as $w_{g} / U_{\infty}=0.08$, causing that the angle of attack of airfoil suddenly increased by about $\alpha=4$ (0.08 radian). Moreover, the real time step was $\Delta t=0.001 \mathrm{~s}$ and dimensionless time $S$ was defined as $S=2 t U_{\infty} / c$. LU decomposition was used to perform implicit iteration and calculation of the quasi-time step and the number of iteration steps was 300 . Through the calculation of 300 iterations of pseudo-time steps, the residual reduced by $3 \sim 4$ orders.

Figure 2 demonstrates the response curve of lift coefficient of airfoil with dimensionless time under different Mach numbers. By comparing calculation results (marked as Cal in Figs. 2 and 3) in this study with those (recorded as Ref) in reference (Parameswaran and Baeder 1997), it can be seen that two groups of results coincided well. This indicated that the calculation method of gust response used in the study was reasonable and feasible. Figure 3 shows the comparisons of calculation results and theoretical piston solutions (shown as Exact in Fig. 3). As demonstrated in Fig. 3, when $M_{\infty}=0.3$ the calculation results were well consistent with the theoretical solutions. However, with the increase of Mach number, nonlinear characteristics of flow were enhanced, thus gradually raising the deviation of calculation results and theoretical solutions.

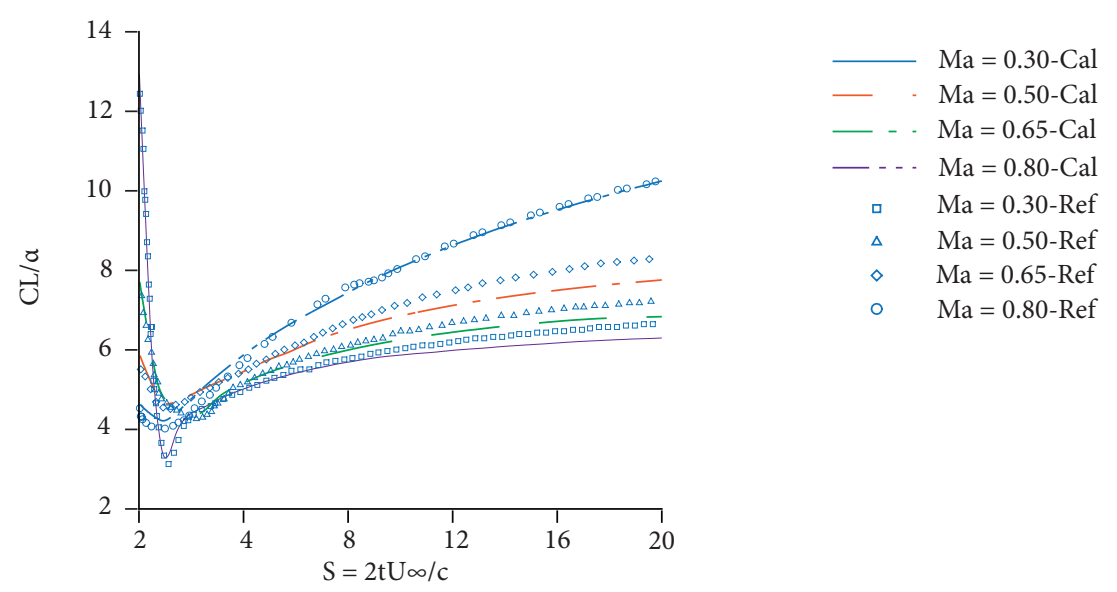

Figure 2. Comparisons of calculation value with reference value of lift response.

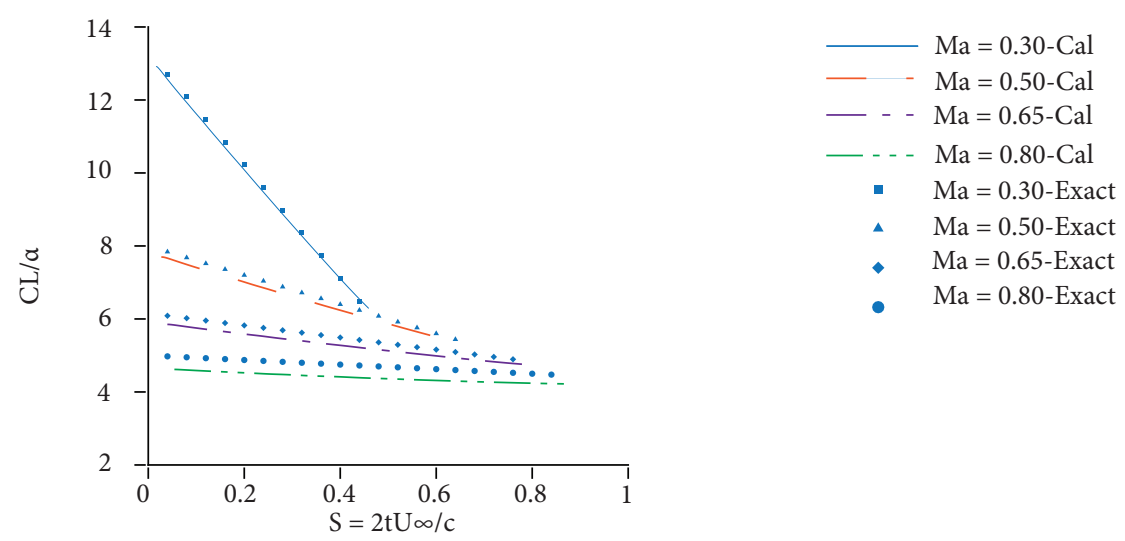

Figure 3. Comparisons of calculation value with theoretical value of lift response. 


\section{RESULTS AND DISCUSSIONS}

The UAV studied in this paper is a high-aspect-ratio profile with $\mathrm{W}$-shaped flying wing configurations without anhedral and torsion angle, as shown in Fig. 4. Moreover, the aspect ratio was $\lambda=11$, the spanwise length was $b=52.12 \mathrm{~m}$ and the distance from the reference point of pitching moment to the head was $8.23 \mathrm{~m}$.

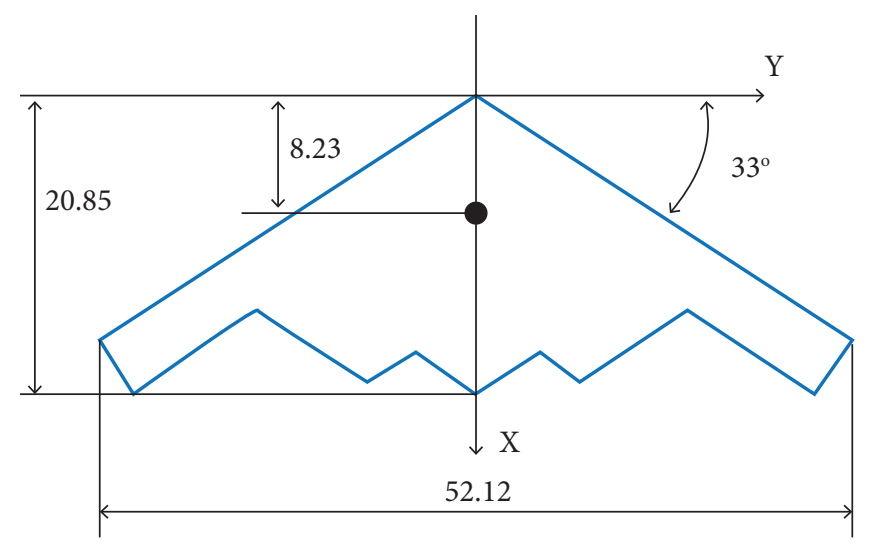

Figure 4. Configuration of the high-aspect-ratio UAV (m).

The gust for calculation is discrete 1-cos gust (Yang and Obayashi 2004), and the gust model is (Eq. 3):

$$
W_{g}=\frac{1}{2} W_{0}[1-\cos (2 \pi x / G G L)]
$$

where: $W_{0}$ indicates the maximum gust velocity and GGL refers to the gust gradient length. In the path perpendicular to the flight of the UAV, gust velocity changed according to the cosine law in gust gradient length and the gust velocity is demonstrated in Fig. 5 .

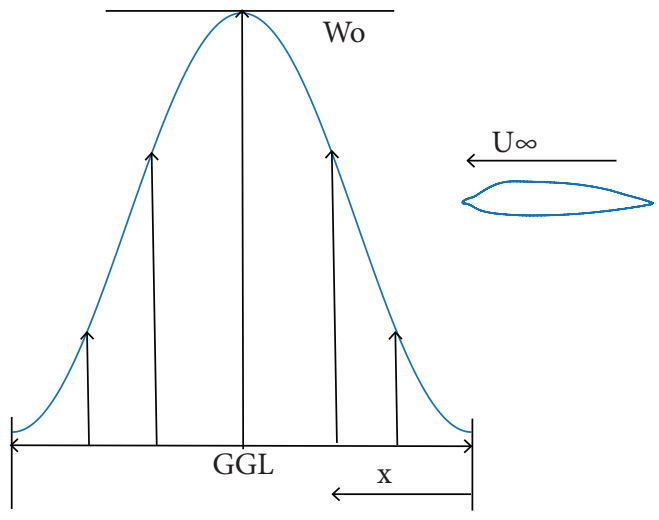

Figure 5. Discrete 1-cos gusts.

Owing to this study has mainly researched the influences of gust response on longitudinal aerodynamic characteristics of the UAV, the half-model of the UAV was used for calculation in order to reduce the calculation amount. Setting the coordinate origin at the head of the UAV. The grid generation procedures were an important issue for simulation, especially for complex flowfield 
and unsteady flow, because the grid quality affected the accuracy and convergence rate of numerical solutions. The computing grid was carried out in the domain whose $X$ direction was 10 times of root chord length of the wing and $Y$ and $Z$ directions were 5 times of the half span. The surface of the UAV was set to the wall boundary, the symmetry plane was set to the symmetrical boundary condition, and the other boundary conditions were all set to the pressure-far-field boundaries. The calculation domain and boundary conditions are shown in Fig. 6.

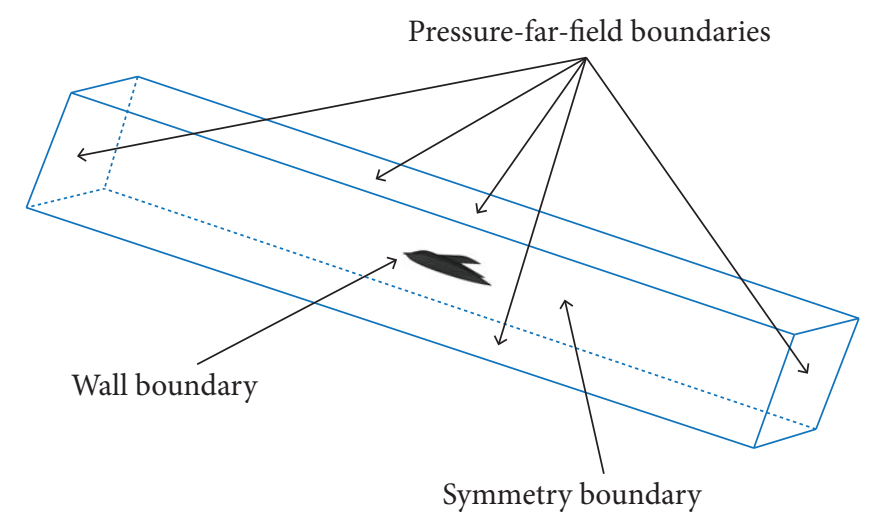

Figure 6. Calculation domain and boundary conditions.

The computational domain of the UAV was divided into 8 Multi-blocks structural grid. A C-H type topological structure around the UAV was used as shown in Fig. 7a, and the number of grid elements was 118973 . The focus of this study was on the lift coefficient and the pitching moment coefficient. The grid near the wall surface was encrypted, and the first grid distance was 0.09 , according to $y+$ almost 10 , as shown in Fig. $7 \mathrm{~b}$. This meshing strategy could avoid the impact of the grid on the calculation results and provide sufficient calculation accuracy. The demand for computing resources was also acceptable (Yang and Ma 2013). In the calculation, Mach number, the angle of attack and Reynolds number were $M_{\infty}=0.08, \alpha=0^{0}$ and $\operatorname{Re}=7.72 \times 10^{8}$, respectively. Moreover, $G G L=25$ and $W_{0}=15.24 \mathrm{~m} / \mathrm{s}$. In the calculation, S-A model was used as turbulence model and the reference length of moment coefficient was mean aerodynamic chord being $b_{A}=12.94 \mathrm{~m}$. The real time step was $\Delta \mathrm{t}=0.001 \mathrm{~s}$ and the dimensionless time $S$ was defined as $S=2 t U_{\infty} / b_{A}$

(a)

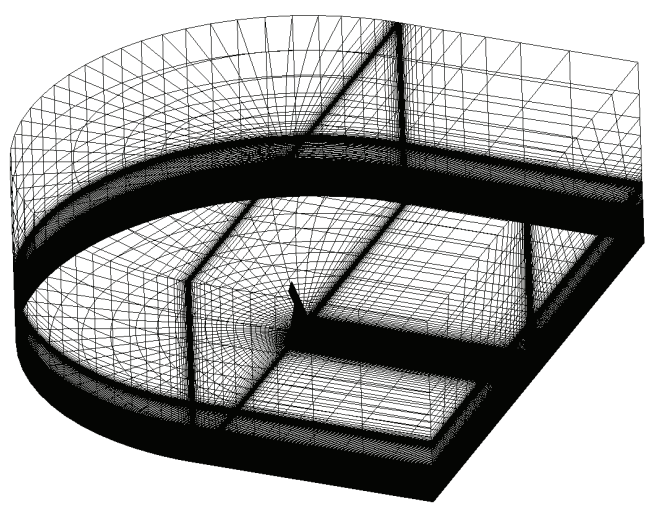

(b)

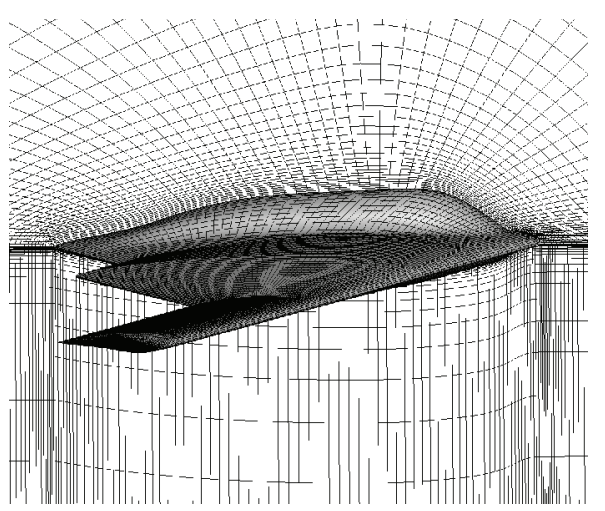

Figure 7. Computing grid. (a) C-H type grid; (b) close view grid around UAVs.

As shown in Fig. 8, under the effects of gusts, lift coefficient changed greatly and gradually recovered to the initial state with the disappearance of gusts. The coefficient of lift increased with the speed of the gust, and the pitching moment coefficient decreased with the speed of the gust. Due to the influences of unsteady hysteresis effects, the response of lift coefficient and pitching moment 
coefficient of the UAV was shown as certain hysteresis. Such hysteresis effects caused certain fluctuations of lift coefficient and pitching moment coefficient after disappearance of gusts.

(a)

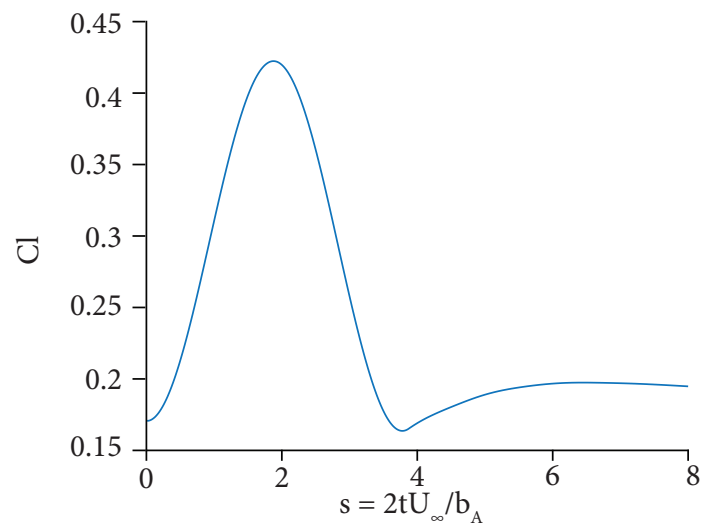

(b)

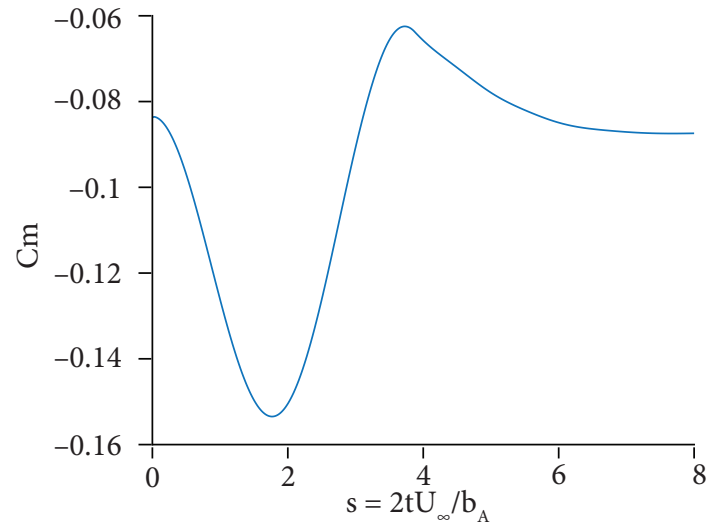

Figure 8. Response curve of longitudinal aerodynamic force lift coefficient of the UAV under 1-cos gusts. (a) Lift coefficient; (b) pitching moment coefficient.

In order to explain the causes for influences of gusts on lift coefficient of the UAV, the pressure coefficient distribution on the $2 y / b=0.69$ spanwise section of the UAV under 1-cos gust was analyzed at different response moments in Fig. 9. It can be seen that as the gust velocity gradually increased, the negative pressure on the upper surface rose, thus increasing lift coefficient. With the further increase of the gust velocity, the negative pressure on the upper surface did not increase anymore while the pressure

(a)

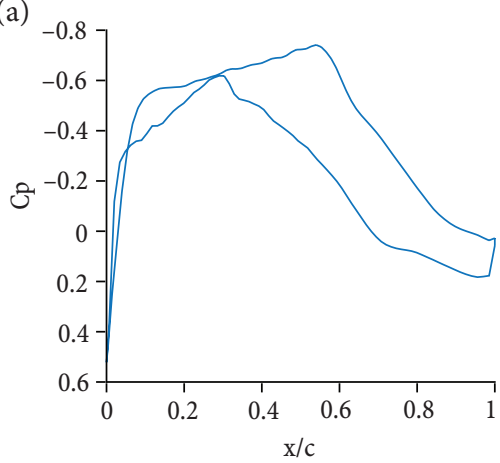

(d)

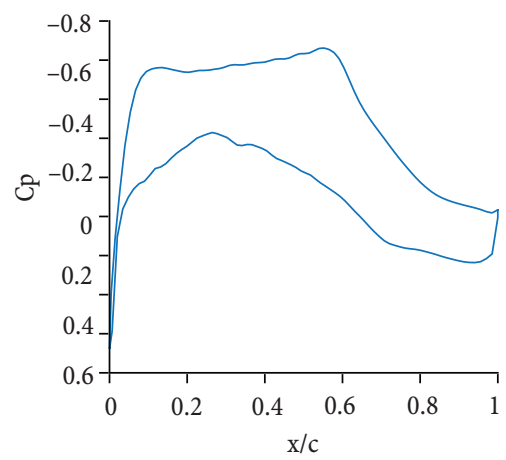

(b)

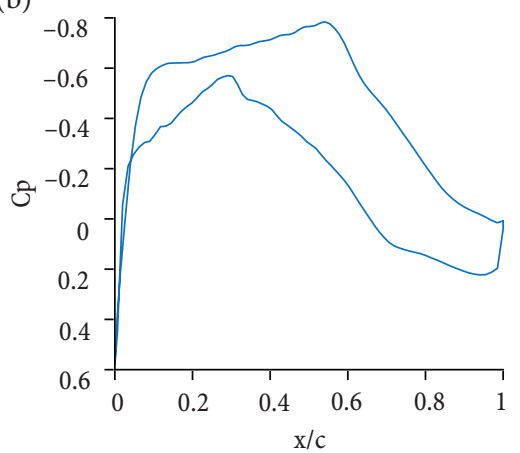

(e)

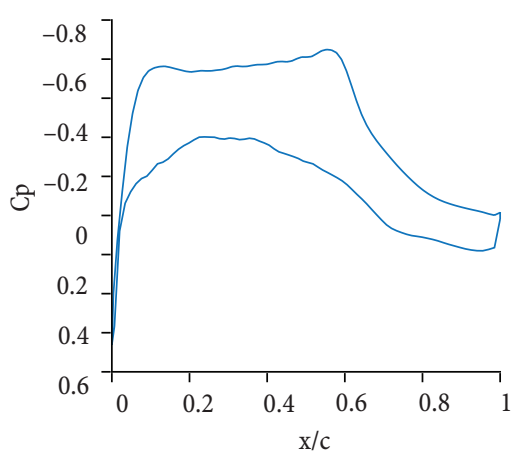

(c)

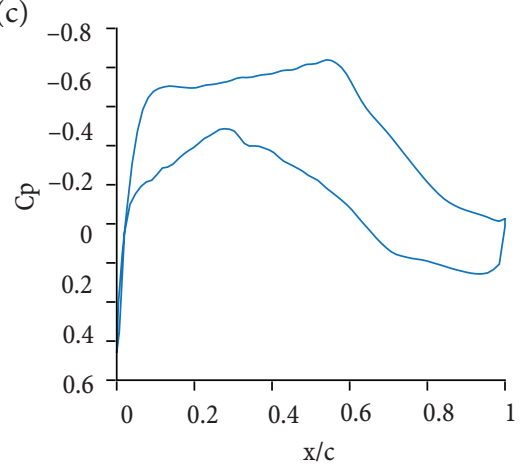

(f)

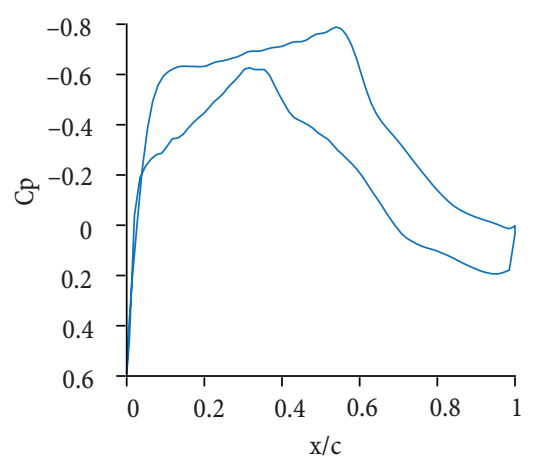

Figure 9. Pressure coefficient distribution on $2 y / b=0.69$ spanwise section of the $U A V$ at different response moments. (a) $s=0.00$; (b) $s=0.84$; (c) $s=1.51$; (d) $s=2.10$; (e) $s=2.77$; (f) $s=8.00$. 
gradient on the lower surface rose, thus further raising the lift coefficient. Until reaching $S=1.96$, the response of lift coefficient was the largest. Then gust velocity gradually decreased and the pressure gradient on the lower surface gradually reduced, which declined lift coefficient. When $S=3.86$, gust velocity reduced to 0 . Because of unsteady hysteresis effects, pressure distribution on the cross section was still affected by disturbances and pressure distribution was basically consistent with the initial state until $S=8.00$.

Figure 10 shows the change curve of pitching moment coefficient of the UAV with lift coefficient under the effects of 1-cos gusts. It can be seen that the changes of pitching moment coefficient with lift coefficient are shown as a hysteresis loop due to unsteady hysteresis effects. With the increase of gust response velocity lift coefficient rose, while the pitching moment coefficient decreased. When the gust velocity reached the extreme value and then began to decrease, the pitching moment coefficient increased while lift coefficient reduced. In the whole gust gradient length, $\Delta c_{m} / \Delta c_{l}<0$ existed, indicating that the UAV was always in the state of static stability under the effects of 1-cos gusts. The influences of 1-cos gusts on static stability margin of the high-aspect-ratio UAV were further analyzed. As demonstrated in Fig. 11, with the increase of gust velocity the static stability margin of the UAV decreased. When the gust velocity reduced from the maximum amplitude, the static stability margin of the UAV rose. Due to the influences of unsteady hysteresis effects, the static stability margin of the UAV was still affected by disturbances and gradually returned to the initial value when gusts disappeared.

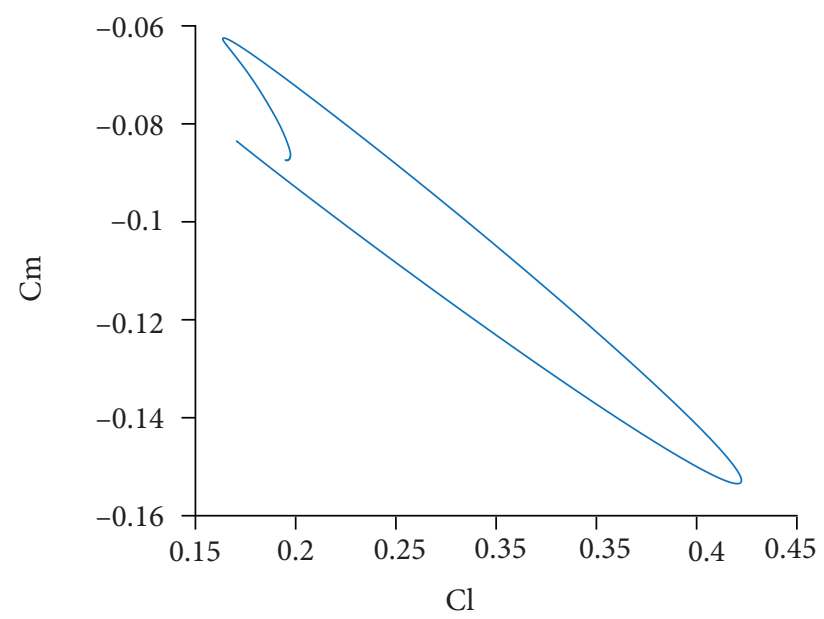

Figure 10. Response curve of pitching moment coefficient of the UAV with lift coefficient under 1-cos gusts.

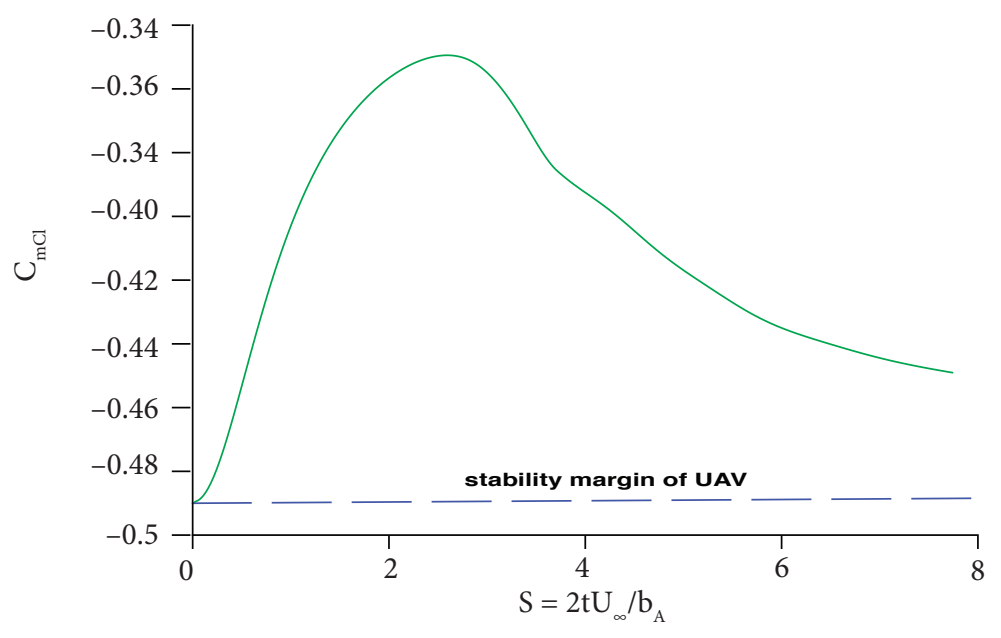

Figure 11. Change curve of static stability margin of the UAV under 1-cos gusts. 


\section{CONCLUSIONS}

(1) By introducing the grid velocity to research the effects of gusts, the numerical simulation method for gust response was established. This method overcame the numerical oscillations and divergence problems in the calculation that may occur due to large gust speeds. In this research, the calculation results coincided with theoretical and reference results, indicating that calculation results were reasonable and reliable.

(2) With the changes in the speed of the 1-cos gusts, the lift and pitching moment coefficients of high-aspect-ratio UAVs changed significantly. The aim of mastering the change laws of longitudinal aerodynamic forces under effects of gusts is to guide the design of gust alleviation system. Longitudinal aerodynamic calculations reveal the UAV was in the state of static stability with the changes of gust speed. However, with the increase of gust velocity, the static stability margin of the UAV reduced, which greatly affected the safe operation of the UAV.

(3) The 1-cos gust response of high-aspect-ratio UAVs with a high Mach number was calculated. However, the Mach number presently used by the high-aspect-ratio UAVs was very low. Therefore, when analyzing the gust response characteristics of highaspect-ratio UAVs at low speed, the mesh division should reduce the dimension of the first layer of the wall boundary and improve the $y+$. At the same time, the low speed pretreatment technique and transfer model were also used in the calculation.

\section{AUTHOR'S CONTRIBUTION}

Conceptualization, Wang J; Methodology, Zhang W and Feng B; Investigation, Ren Z and Zhao Q; Writing - Original Draft, Wang J and Zhang W; Writing - Review and Editing, Wang J; Funding Acquisition, Ren Z; Resources, Zhao Q and Ren Z; Supervision, Wang J.

\section{FUNDING}

National Natural Science Foundation of China

Grant no. 51605270

Natural Science Research Project of Shaanxi Provinc

Grant no. 2016JM1030

Science Technology Department Key Project of Shaanxi Province

Grant no. 2017ZDXM-GY-138

Industrial Science and Technology Research Project of Shaanxi Province

Grant no. 2016GY-050

\section{REFERENCES}

Gennaretti M, Mastroddi F (2004) Study of reduced-order models for gust-response analysis of flexible wings. Journal of Aircraft 41(2):304-313. https://doi.org/10.2514/1.9325

Gilmanov A, Sotiropoulos F (2005) A hybrid cartesian/immersed boundary method for simulating flows with 3d, geometrically complex, moving bodies. Journal of Computational Physics 207(2):457-492. https://doi.org/10.1016/i.jcp.2005.01.020

Gong CY, Bao WM, Liu J, Tang GT, Jiang YE (2016) An efficient wavefront parallel algorithm for structured three dimensional LU-SGS. Computers \& Fluids 134-135:23-30. https://doi.org/10.1016/i.compfluid.2016.05.008

Gu L, Lu ZL, Guo TQ (2013) Gust response analysis of free elastic wing in the transonic flight regime. Journal of Vibration Engineering 26(5):700-706. https://doi.org/10.16385/j.cnki.issn.1004-4523.2013.05.017 
Guo S, Jing ZW, Li H, Lei WT, He YY (2017) Gust response and body freedom flutter of a flying-wing aircraft with a passive gust alleviation device. Aerospace Science and Technology 70:277-285. https://doi.org/10.1016/j.ast.2017.08.008

Liu Y, Xie CC, Yang C, Cheng JL (2016) Gust response analysis and wind tunnel test for a high-aspect ratio wing. Chinese Journal of Aeronautics 29(01):91-103. https://doi.org/10.1016/j.cja.2015.12.013

Orang AA (2014) Genuinely characteristic-based scheme for the incompressible turbulent flows. Computers \& Fluids 103:175-185. https://doi.org/10.1016/i.compfluid.2014.07.015

Parameswaran V, Baeder JD (1997) Indicial aerodynamics in compressible flow-direct computational fluid dynamic calculations. Journal of Aircraft 34(1):131-133. https://doi.org/10.2514/2.2146

Patil MJ, Taylor DJ (2006) Gust response of highly flexible aircraft. Presented at: 47th AIAA/ASME/ASCE/AHS/ASC Structures, Structural Dynamics and Materials Conference; Newport, USA. https://doi.org/10.2514/6.2006-1638

Shao K, Wu ZG, Yang C, Chen L (2010) Theoretical and experimental study of gust response alleviation using neuro-fuzzy control law for a flexible wing model. Chinese Journal of Aeronautics 23(3):290-297. https://doi.org/10.1016/S1000-9361(09)60218-1

Singh R, Baeder JD (1997) Direct calculation of three-dimensional indicial lift response using computational fluid dynamics. Journal of Aircraft 34(4):465-471. https://doi.org/10.2514/2.2214

Song WP, Yang, Y, Qiao ZD, Pahlke K (1999) Unsteady N-S calculations using a dual-time stepping method for airfoil oscillating at high angle of attack. Acta Aerodynamica Sinica 17(4):466-471. https://doi.org/10.3969/i.issn.0258-1825.1999.04.016

Tang D, Grasch A, Dowell EH (2010) Gust response for flexibly suspended high-aspect ratio wings. AlAA Journal 48(10):2430-2444. https://doi.org/10.2514/1.J050309

Van Albada GD, Van Leer B, Roberts Jr WW (1982) A comparative study of computational methods in cosmic gas dynamics. Astronomy and Astrophysics 108(1)76-84.

Wang JL (2011) Numerical study on unsteady aerodynamics and aeroelasticity of flying-wing UAV (PhD Dissertation). Xi'an: Northwestern Polytechnical University. In Chinese.

Wu ZG, Chen L, Yang C, Tang CH (2010) Gust response modeling and alleviation scheme design for an elastic aircraft. Science China Technological Sciences 53(11):3110-3118. https://doi.org/10.1007/s11431-010-4141-y

Yang GW, Obayashi S (2004) Numerical analyses of discrete gust response for an aircraft. Journal of Aircraft 41(6):1353-1359. https://doi.org/10.2514/1.2531

Yang MQ, Ma DL (2013) Effect of asymmetric ground effect on stabilitiy of tailless aircraft. Journal of Aerospace Power 28(11):25532560. https://doi.org/10.13224/i.cnki.jasp.2013.11.021

Zaide A, Raveh D (2006) Numerical simulation and reduced-order modeling of airfoil gust response. AlAA 44(8):1826-1834. https:// doi.org/10.2514/1.16995

Zhan H, Qian WQ (2007) Numerical simulation of gust response for thin airfoil. Acta Aeronautica Et Astronautica Sinica 28(3):527-530.

Zhang WW, Ye ZY, Yang Y, Shi AM (2009) Gust response analysis using cfd-based reduced order models. Presented at: 47th AIAA Aerospace Sciences Meeting including The New Horizons Forum and Aerospace Exposition; Orlando, USA. https://doi.org/10.2514/6.2009-895 